\title{
Cooperativity, priming, and 3-D surface detection from optic flow
}

\author{
PAUL ATCHLEY, GEORGE J. ANDERSEN, and A. PAIGE WUESTEFELD \\ University of California, Riverside, California
}

\begin{abstract}
Three experiments were conducted to determine whether the mechanisms responsible for the detection of three-dimensional (3-D) surfaces from optic flow operate in a cooperative manner. The first experiment was conducted to determine whether a hysteresis effect occurs for 3-D surface detection from optic flow. The results of the first experiment demonstrated a hysteresis effect with lower thresholds occurring for decreasing texture density than for increasing texture density. The second experiment used a priming methodology to determine whether this form of cooperativity was based on preactivation of shear detectors or preactivation of 2-D motion detectors. The results suggest that only shear detectors were primed. The third experiment utilized a similar methodology to determine whether a surface representation would produce a priming effect. We found no evidence that the priming effect found in the second experiment was the result of preactivation of a generic representation of the test stimuli. The results of the experiments, considered together, suggest priming of the mechanisms responsible for recovering shear.
\end{abstract}

Optic flow (Gibson, 1966; see also Helmholtz, $1867 /$ 1962) provides information useful for the recovery of three-dimensional (3-D) shape. Previous research has demonstrated that optic flow can be used to recover surface slant and orientation (e.g., Braunstein \& Andersen, 1981; Braunstein \& Payne, 1969; Braunstein \& Tittle, 1988), recover the shape (e.g., Rogers \& Graham, 1979; Rogers \& Rogers, 1992) and curvature of a surface (e.g., Norman \& Lappin, 1992; Todd, 1984), and detect the rigidity of surfaces (e.g., Braunstein, Hoffman, \& Pollick, 1990; De Bruyn \& Orban, 1990; Todd, 1984). In addition to empirical research on optic flow, there has been considerable interest in formal analyses of optic flow for the recovery of shape and depth information (Koenderink, 1986; Koenderink \& van Doorn, 1977; Longuet-Higgins \& Prazdny, 1980; Verri, Girosi, \& Torre, 1990). For example, Koenderink and his colleagues (Koenderink, 1986; Koenderink \& van Doorn, 1977) mathematically demonstrated that deformation of the flow field could be used for 3-D shape recovery, and they proposed a set of simple mechanisms (see Figure 1) that could be used for recovering the shear components of deformation.

In addition to 3-D shape recovery, optic flow also provides information for the detection of 3-D surfaces. Previous research on surface detection from optic flow (Andersen, 1996) has proposed that the detection of 3-D surfaces could be performed by a lattice of shear detectors. Andersen (1996) proposed an analysis based on the summed value of the two shear components (Koenderink,

This research was partially supported by an AFOSR Fellowship to the first author, NSF Grant SBR 95-10431, and NIH Grant AG1341901. Correspondence should be addressed to G. J. Andersen, Department of Psychology, University of California, Riverside, CA 92521 (e-mail; andersen $(a$ citrus.urc.edu).
1986) of deformation determined by two shear detectors (see Figure 1). The analysis of smoothness using the two shear components of the velocity field was derived by calculating an average output of the four receptive fields of the two shear components (see Figure 1). Analyses of the shear components of deformation using single sinewave and complex sine-wave corrugated surfaces yielded $d^{\prime}$ values that were significantly correlated with the mean $d^{\prime}$ values derived from human observers.

Although a considerable body of research has examined 3-D shape and surface recovery from optic flow, little research has been conducted on the cooperativity of the mechanisms responsible for surface and shape recovery. Cooperativity is a method proposed in both physical and neural systems for combining common information over space and/or time. In a cooperative system, neural mechanisms tuned to similar values are linked nonlinearly in an excitatory fashion. Dissimilar mechanisms are linked in an inhibitory fashion. This allows the system to extract a common signal from a broad number of weakly correlated subsignals and to remain in this state despite variations in the subsignal characteristics over time (Feldman \& Ballard, 1982).

Cooperativity in a network of neural mechanisms can occur either spatially or temporally. One method that has been used to determine spatial or temporal cooperativity is a hysteresis paradigm. In this method, subjects are shown displays in which the critical information of interest (e.g., the signal-to-noise ratio of disparity in the case of stereopsis; the signal-to-noise ratio of motion direction in the case of motion) is either increased or decreased. This paradigm has been used to assess cooperativity of mechanisms for fusion in binocular disparity (Chang \& Julesz, 1984; Fender \& Julesz, 1967; Julesz, 1971, 1983) and motion coherence (Chang \& Julesz, 1984; 

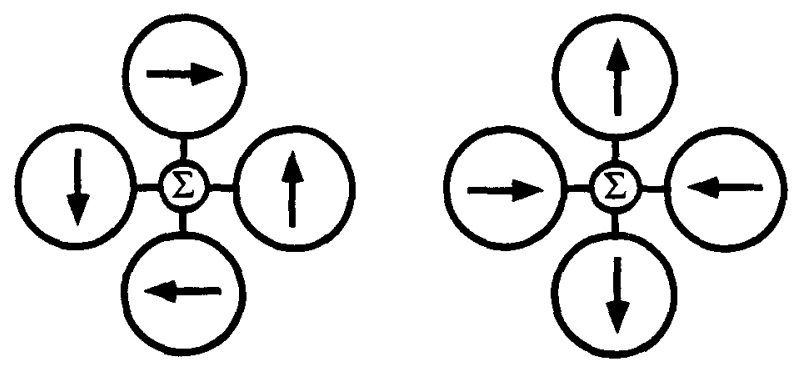

Figure 1. Illustration of the two shear detectors, pmoped by Koenderink and van Doorn (1977), for recovering the deformation of the flow field.

Nawrot \& Sekuler, 1990; Williams \& Phillips, 1987; Williams, Phillips, \& Sekuler, 1986). For example, Fender and Julesz (1967) presented observers with disparity displays in which the magnitude of disparity either increased (from $0^{\prime}$ to $100^{\prime}$ of arc) or decreased (from 100' to $0^{\prime}$ of arc) over time. For increasing disparity trials, subjects were asked to indicate when the display could no longer be fused. For decreasing disparity trials, subjects were asked to indicate when fusion occurred. The results indicated that the diplopia threshold for an unfused stimulus that is slowly brought into correspondence is smaller than the diplopia threshold for an initially fused stimulus that is slowly increased in disparity. This phenomenon has been referred to as a hysteresis effect-a system bias to remain in a current state.

Similar results have also been obtained for 2-D motion tasks (Chang \& Julesz, 1984; Nawrot \& Sekuler, 1990; Williams \& Phillips, 1987; Williams et al., 1986). Chang and Julesz demonstrated spatial cooperativity for motion detectors by showing that apparent motion induced by random-dot cinematograms can induce a similar percept in neighboring regions of random motion. Williams et al. also demonstrated that the motion perception system has temporally cooperative mechanisms. In their experiments, they used random-dot cinematograms in which a correlated motion signal either increased from a value that was not perceived as coherent motion or decreased from a value that was perceived as coherent motion. They found that the number of correlated points necessary to go from a perception of incoherent motion to a perception of coherent motion was higher than in the opposite case.

A second methodology that has been used to assess the nature of cooperative processing is a priming paradigm. In a priming paradigm, subjects are presented with a stimulus (referred to as the prime) prior to the presentation of a target stimulus. If the prime contains information important for the task under investigation, then improved performance will occur. Evidence of a priming effect is also evidence of temporal facilitation or cooperativity since the presentation of the prime preactivates or primes the mechanisms important for the task and thus results in improved performance. Priming paradigms have been used in a variety of contexts, including studies on memory (e.g., McNamara, 1992; Reingold \& Goshen-Gottstein, 1996), visual attention (e.g., Fox, 1995; May, Kane, \& Hasher, 1995), object recognition (Biederman \& Cooper, 1991; Lawson, Humphreys, \& Watson, 1994), psycholinguistics (e.g., Tenpenny, 1995), and categorization (e.g., Chiappe \& MacLeod, 1995).

The purpose of the present research was twofold. First, we were interested in determining whether evidence of cooperativity could be observed for the detection of 3-D surfaces from optic flow. Given that previous studies (Nawrot \& Sekuler, 1990; Williams \& Phillips, 1987; Williams et al., 1986) have demonstrated cooperative processing for 2-D motion recovery, an important question is whether cooperativity is also important for mechanisms responsible for the recovery of 3-D surfaces from optic flow. We examined this issue by using a hysteresis paradigm in Experiment 1 and a priming paradigm in Experiments 2 and 3. Second, we were interested in the level at which cooperativity could occur. Three possible levels in which cooperativity could occur were examined. One level in which cooperativity could occur is the mechanisms responsible for the recovery, of local shear information. A second level is the initial recovery of the $2-D$ velocities of the display. Finally, a third level is the encoding of a generic surface representation. We examined these three possibilities in Experiments 2 and 3 by presenting subjects with three different primes: a flow field of the target surface, a randomized version of the flow field of the target surface, and a flow field of the target surface undergoing a different motion.

\section{EXPERIMENT 1}

Experiment I was designed to determine whether evidence of a hysteresis effect occurs for the detection of 3-D surfaces from optic flow. In this experiment, the subjects were shown optic flow displays with either an increasing or a decreasing texture density. In the increasing case, the trial began with very few dots and was gradually increased until a 3-D surface was perceived. In the decreasing case, the 3-D surface was readily apparent at the beginning of the trial, and dots were removed until a 3-D surface was not perceived. If a hysteresis effect occurs for the detection of 3-D surfaces from optic flow, then thresholds should be lower for the decreasing texture condition than for the increasing texture condition.

\section{Method}

Subjects. The subjects were 8 psychology graduate students at the University of California, Riverside, who were naive with regard to the purpose of the experiment. The subjects were paid for their participation. All had normal or corrected-to-normal vision.

Apparatus. The stimuli were generated and displayed on an Evans and Sutherland ESV 50 Graphics Workstation and an SGI Indigo2 Extreme graphics workstation. The resolution of both systems was $1,280 \times 1,024$, and the frame rate was $30 \mathrm{~Hz}$. The velocities, pixel size $\left(1.67^{\prime}\right.$ of $\left.\operatorname{arc}^{2}\right)$, and frame rate $(30 \mathrm{~Hz})$ were equivalent for both systems. In addition, the displays of both systems were perceptually matched for brightness. The displays were viewed monocularly at a distance of $1.5 \mathrm{~m}$ through a viewing tube 
arrangement that restricted the field of view to a circular area $11.0^{\circ}$ in diameter.

Design. The experiment employed a $2 \times 2 \times 2$ within-subjects design. The independent variables were the spatial frequency of the sinusoidal surface ( 0.277 or $0.455 \mathrm{cycles} / \mathrm{deg}$ of visual angle), the amplitude or velocity ratio of the slowest and fastest point (1.20 or 1.43), and the direction of change in texture density (increasing or decreasing)

Stimuli. The stimuli were computer-generated displays that simulated points positioned on a 3-D surface. The surface displays were generated in the following manner: Points were randomly positioned in the image and translated along the horizontal axis. Velocities were assigned to the points according to a sine function that varied along the vertical axis. The position change of a point in the image per frame (i) was determined using the following equation:

$$
i=a \sin \left(f a y / y_{\max }\right)+b
$$

where $a$ is the amplitude of the surface, $b$ is the minimum horizontal motion per frame, $f$ is the frequency of the surface, $y$ is the vertical coordinate (in pixel units) of the point, and $y_{\max }$ is the maximum $y$ value. This simulated a surface in depth that was corrugated along the vertical axis.

The displays subtended a $11.42^{\circ} \times 14.27^{\circ}$ rectangular region. (Actual viewing size was restricted by the viewing tube.) The size of each point was $1.67^{\prime}$ of $\mathrm{arc}^{2}$. The minimum velocity for each display was $3.23 \% \mathrm{sec}$. The maximum velocity for a display was determined by the amplitude value: $3.88 \% \mathrm{sec}$ for an amplitude value of 1.20 , and $4.62 \% \mathrm{sec}$ for an amplitude value of 1.43 . The display duration varied (see below).

The simulated surfaces repeatedly translated from left to right and from right to left. The duration of each left-to-right/right-to-left oscillation was $3 \mathrm{sec}$. Increasing density trials started with a density of $0.122 \mathrm{dots} / \mathrm{deg}^{2}$ (number of dots). Density increased at a rate of $0.122 \mathrm{dots} / \mathrm{deg}^{2} / \mathrm{sec}$ until a response was made or the maximum density of 6.13 dots $/ \mathrm{deg}^{2}$ was reached. The decreasing density trials were similar, with the exception that the starting density was $6.13 \mathrm{dots} / \mathrm{deg}^{2}$, and dots were removed at a rate of $0.122 \mathrm{dots} / \mathrm{deg}^{2 /}$ $\mathrm{sec}$ until a keypress or a density of $0.00 \mathrm{dots} / \mathrm{deg}^{2}$ was reached.

Procedure. Each subject participated individually. The subjects were informed that they would see a series of displays containing moving white dots. On some of the trials, the display would start with very few dots, and, over time, more dots were added (increasing density). On other trials, the display would start with a large number of dots, with dots removed over time (decreasing density) (see Figure 2). In the former case, the subjects' task was to press a key when they saw a "compelling" impression of a 3-D corrugated surface. In the latter case, the subjects' task was to press a key when the display no longer appeared as a "compelling" 3-D corrugated surface. For each cell in the design, there were 30 trials.

The experiment took place in four 1-h blocks run over a period of 1 week. Each session was blocked according to frequency and amplitude, with both increasing and decreasing trials run sequentially during the same block. Order was counterbalanced for all variables. When a key was pressed, the current density level was recorded, and a new trial was started.

\section{Results and Discussion}

All subjects reported a compelling perception of depth from the displays, with all displays producing a compelling impression of a 3-D surface at some point during the trial. Data were analyzed using a $2 \times 2 \times 2$ withinsubjects analysis of variance (ANOVA). For each surface type (frequency and amplitude) and direction, an average density value when the subjects responded was calculated for each subject (the mean of the 30 individual tri- als), for a total of 8 data points per condition. The main effect of frequency was significant $[F(1,7)=24.33, p<$ $.01]$. The subjects reported a perception of the lowfrequency corrugation surfaces at a lower texture density $\left(0.55 \mathrm{dots} / \mathrm{deg}^{2}\right)$ than the high-frequency corrugation surfaces $\left(1.01 \mathrm{dots} / \mathrm{deg}^{2}\right)$. There was also a main effect of direction $[F(1,7)=10.06, p<.05]$. On the increasingtexture-density trials, the subjects did not report seeing a 3-D corrugated surface until an average texture density of $0.96 \mathrm{dots} / \mathrm{deg}^{2}$. However, when the trial started with a high texture density, the subjects did not report a loss of perception of a 3-D corrugated surface until an average texture density of $0.61 \mathrm{dots} / \mathrm{deg}^{2}$. This finding indicates that, for descending trials, the subjects perceived a 3-D surface with $36 \%$ less texture density. No other effects or interactions were significant.

The finding of lower detection thresholds for lowfrequency surfaces is similar to the results reported in previous studies (Andersen, 1996; Andersen \& Wuestefeld, 1993). The important result of the present experiment is that the subjects had lower thresholds for decreasingdensity trials than for increasing-density trials. These results are similar to the results of other studies concerned with hysteresis and stereoscopic fusion (Fender \& Julesz, 1967), and they indicate that a hysteresis effect was observed for the detection of 3-D surfaces from optic flow.

Why would subjects report perceiving a 3-D surface at lower density thresholds if they are previously shown the optic flow field that defines the 3-D surface? One possible interpretation is that preexposure to the velocities that define the 3-D surface activates the velocity-tuned motion detectors that allow the subject to detect the surface. This possibility suggests that preexposure to the family of velocities that define the surface activates or primes the 2-D motion detectors important in detecting the surface.

A second possible interpretation is that the preexposure of the optic flow field activates or primes the mechanisms important for 3-D surface detection from optic flow. Koenderink and van Doorn (1977; see also Koenderink, 1986; Longuet-Higgins \& Prazdny, 1980; Verri et al., 1990) have mathematically shown that the deformation of the flow field can be used for 3-D shape recovery. Deformation is defined as

$$
\operatorname{def}=\left\{(\operatorname{def} 1)^{2}+(\operatorname{def} 2)^{2}\right\} 1 / 2,
$$

where def 1 and def 2 are the two shear components of deformation. The two shear components specify the local slant and tilt of the surface and are mathematically defined as

$$
\begin{aligned}
& \operatorname{def} 1=\frac{\partial V_{x}}{\partial_{x}}-\frac{\partial V_{y}}{\partial_{y}}, \\
& \operatorname{def} 2=\frac{\partial V_{x}}{\partial_{x}}+\frac{\partial V_{y}}{\partial_{y}},
\end{aligned}
$$

where $V_{x}$ and $V_{y}$ refer to the horizontal and vertical velocity component, respectively. Koenderink and van Doorn (1977) proposed that the visual system has two special- 


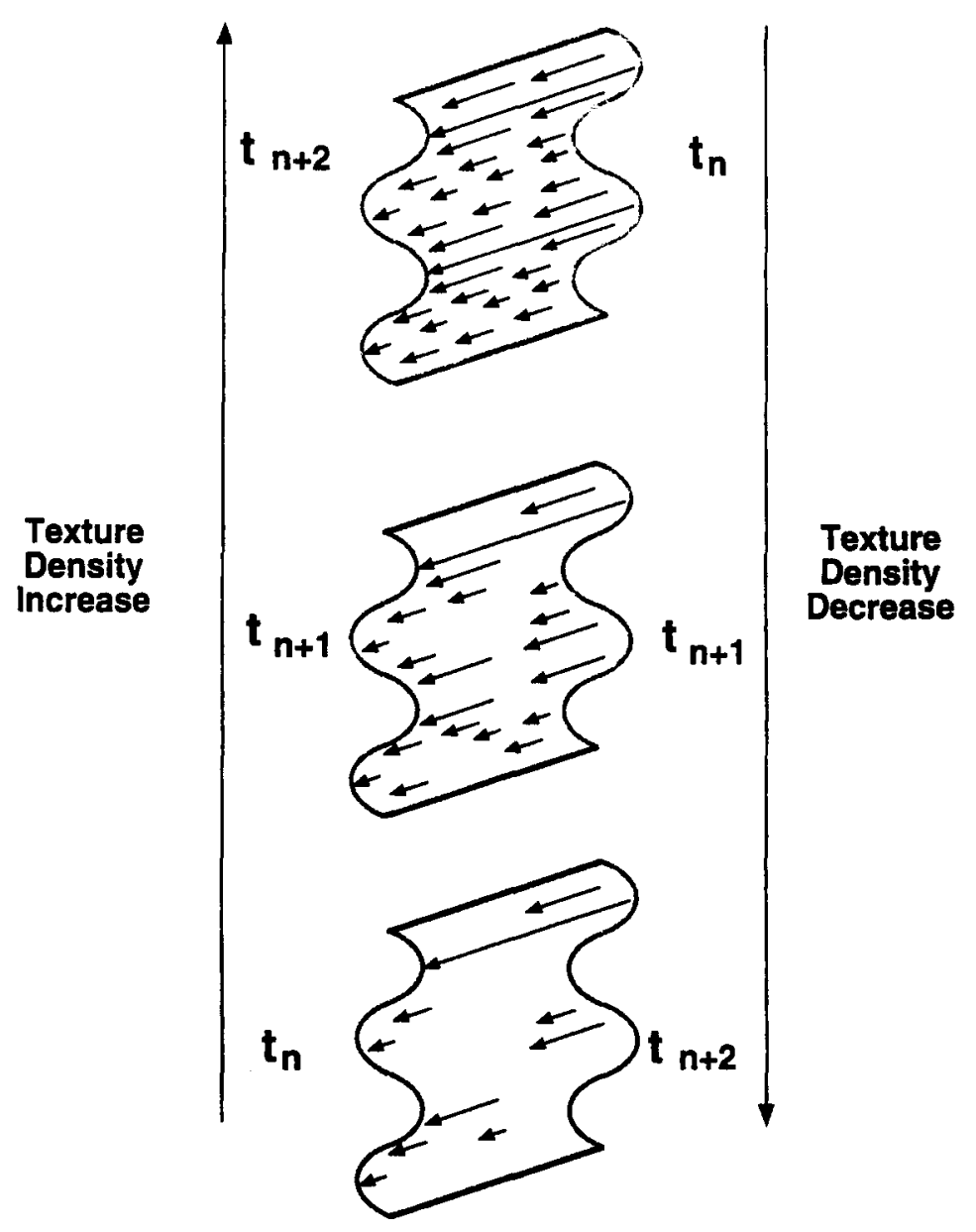

Figure 2. A time line for the increasing and decreasing texture trials in Experiment 1 , where $t_{n}$ is start of the trial and $t_{n+1,2}$ are subsequent points in time during the same trial.

ized detectors responsible for the recovery of the two shear components of deformation (see Figure 1). Each detector outputs a summed value from the simultaneous sampling of velocity, in a specified direction, in four local regions of the visual field. Recently, we have shown how a model based on a two-layer lattice of shear detectors could account for the detection of 3-D surfaces from optic flow by human observers (Andersen, 1996; Andersen \& Atchley, 1995). In addition, neurophysiological studies (Lagae, Maes, Raiguel, Xaio, \& Orban, 1994) have found cells in area MST that respond selectively to the shear components proposed by Koenderink and van Doorn.

\section{EXPERIMENT 2}

In Experiment 2, we employed a priming paradigm to determine whether the mechanisms responsible for 3-D surface detection operate in a cooperative manner. In addition, we examined two possible sources of information of priming. One possibility is that there is priming of mechanisms involved in recovering the family of veloc- ities in the display. The second possibility is that there is priming of the two shear detectors used for recovering the deformation component of the flow field for 3-D surface detection.

The subjects were presented with a priming stimulus followed by two adjacently presented flow fields (see Figure 3 ). One flow field simulated a 3-D corrugated surface similar to the displays examined in Experiment 1. The other flow field consisted of a randomized version of the 3-D surface flow field in which the velocities were randomly repositioned in the display. Both displays had identical sets of velocities. However, our previous research examining a shear detector model has demonstrated that the output of shear detectors is near zero for randomized flow fields but is greater than zero for flow fields simulating a 3-D corrugated surface.

The present experiment used a two-alternative forcedchoice (2AFC) task in which the subjects were asked to identify which of the two adjacently presented flow fields simulated a 3-D corrugated surface. The flow fields presented in the $2 \mathrm{AFC}$ task contained density levels at or 
below threshold for detecting similar corrugated surfaces (Andersen, 1996; Andersen \& Atchley, 1995). One of three different primes preceded the 2AFC displays: no prime (a blank display); a flow field simulating the 3-D corrugated surface to be detected on that trial; or a randomized flow field in which the velocities of the 3-D corrugated surface flow field were randomly repositioned in the image (see Figure 3 ). If no priming occurs, then detection performance should be equivalent regardless of the type of prime presented. If priming is the result of the individual velocities in the flow field, then lower thresholds should occur for the randomized flow field prime. If priming is the result of the shear components of the display, then lower thresholds should occur for the 3-D corrugated surface prime, relative to the random or noprime condition.

\section{Method}

Subjects. The subjects were 8 psychology graduate and undergraduate students at the University of California, Riverside, who were naive with regard to the purpose of the experiment. The subjects were paid for their participation. All had normal or correctedto-normal vision.

Apparatus. The displays were generated and presented on an SGI Indigo2 Extreme. The monitor resolution was $1,280 \times 1,024$, and the frame rate was $72 \mathrm{~Hz}$. The displays were viewed monocularly using an eyepatch at a distance of $1 \mathrm{~m}$.

Design. The experiment employed a $2 \times 2 \times 2 \times 3$ withinsubjects design. The independent variables were the spatial frequency of the sinusoidal surface $(0.184$ or $0.368 \mathrm{cycles} / \mathrm{deg}$ of visual angle), the amplitude or simulated depth of the surfaces $(1.20$ or 1.43), the density of the target display $\left(0.16\right.$ or $\left.0.32 \mathrm{dots} / \mathrm{deg}^{2}\right)$, and the type of prime (3-D corrugated surface, random, or no prime).

Stimuli. The stimuli were computer displays generated in the same manner as those used in Experiment 1. The displays subtended a $11.22^{\circ} \times 11.22^{\circ}$ rectangular region. The size of each point was $2.00^{\prime}$ of $\operatorname{arc}^{2}$. The minimum velocity for any display was $2.15 \%$ sec. The maximum velocity for a display was determined by the amplitude value: $2.58 \% \mathrm{sec}$ for an amplitude of 1.20 , and $3.07 \% \mathrm{sec}$ for an amplitude of 1.43 .

There were two phases to each trial: a prime phase and a target phase (see Figure 4). For each trial, the same frequency and amplitude were used to generate all displays. The prime phase had a duration of $4 \mathrm{sec}$, and it consisted of a 3-D surface prime (a flow field simulated a $3-D$ corrugated surface), a random prime (a flow field in which the velocities of the 3-D corrugated surface were randomly repositioned in the image), or no prime. The surface and motion primes had a density of $7.95 \mathrm{dots} / \mathrm{deg}^{2}$ and either translated to the left or to the right in accordance with the direction of motion of the target phase displays. The interval between the prime and target phase was $1 \mathrm{sec}$. The target phase consisted of rectangular regions side by side of the same size $\left(11.22^{\circ} \times 11.22^{\circ}\right.$ of visual angle) as the region in the prime phase, separated by $1^{\circ}$ of visual angle. One region contained a flow field simulating a 3-D corrugated surface, whereas the other region contained a flow field in which the velocities of the 3-D corrugated surface flow field were randomly repositioned in the display. Direction of horizontal translation and the location of the surface/random volume (left flow field or right flow field) were randomized from trial to trial. For each trial, each region of the two target displays had the same density (either 0.16 or $0.32 \mathrm{dots} / \mathrm{deg}^{2}$ ). Target phase display duration was $1 \mathrm{sec}$.

Procedure. Each subject participated individually. The subjects were informed that they would be participating in a surface detec-

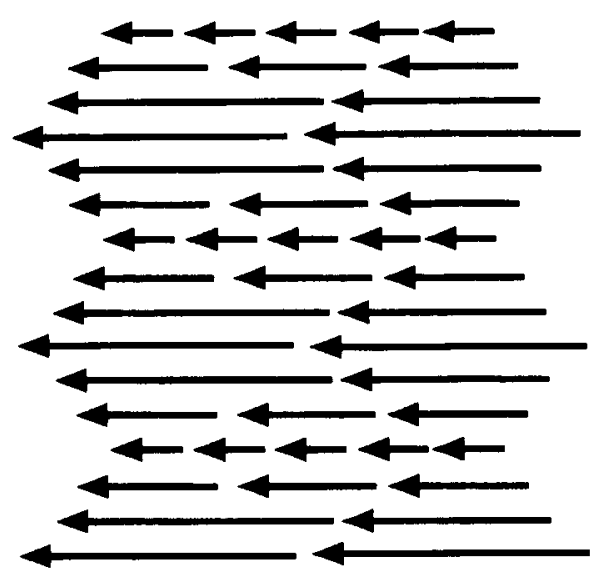

Surface Condition

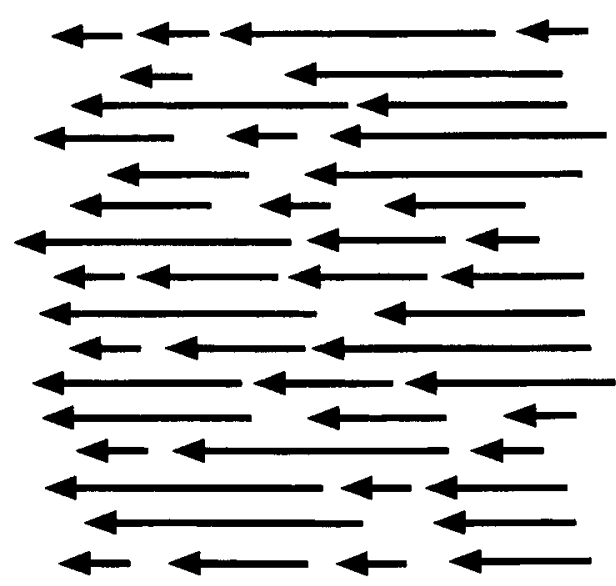

Random Condition

Figure 3. Examples of two optic flow fields utilized in the present research. The surface display is the velocity field produced by a perspective horizontal translation of a 3-D surface corrugated in depth according to a sine function. The random display is a randomized version of the flow field used for the surface display. The velocity vectors are regularly spaced along the axis for the purposes of illustration. In the present experiments, the velocities were randomly positioned in the image.

tion experiment. They were told that they would be shown all of the surfaces they would be asked to detect, prior to the start of the experimental trials. They were then instructed that each experimental trial would consist of two phases. The first phase would show the surface they would be asked to detect, a group of moving dots. or nothing. The second phase was the detection phase in which they were asked to indicate (by pressing the left or the right arrow key on the keyboard) which of two adjacent displays simulated one of the four 3-D corrugated surfaces. The subjects were told that the texture density would be much lower during the target phase than during the prime phase.

There were three experimental sessions, each lasting about $1 \mathrm{~h}$. The sessions were run in a darkened room on separate days. The sessions were blocked by prime type, with the session order ran- 


\section{Phase 1: Prime}
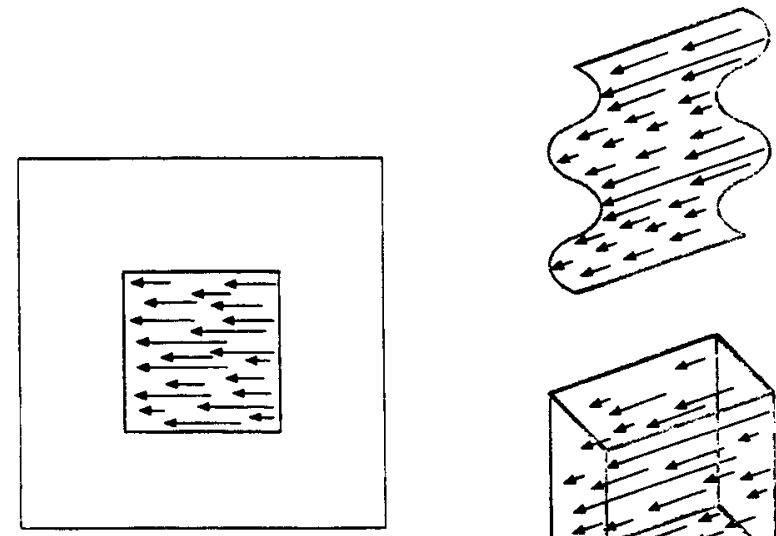

1) Surface Prime

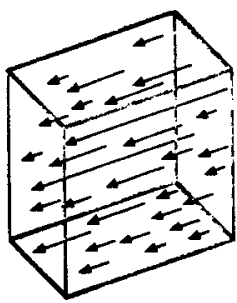

2) Motion Prime

"get ready...

3) No prime

\section{Phase 2: Test}
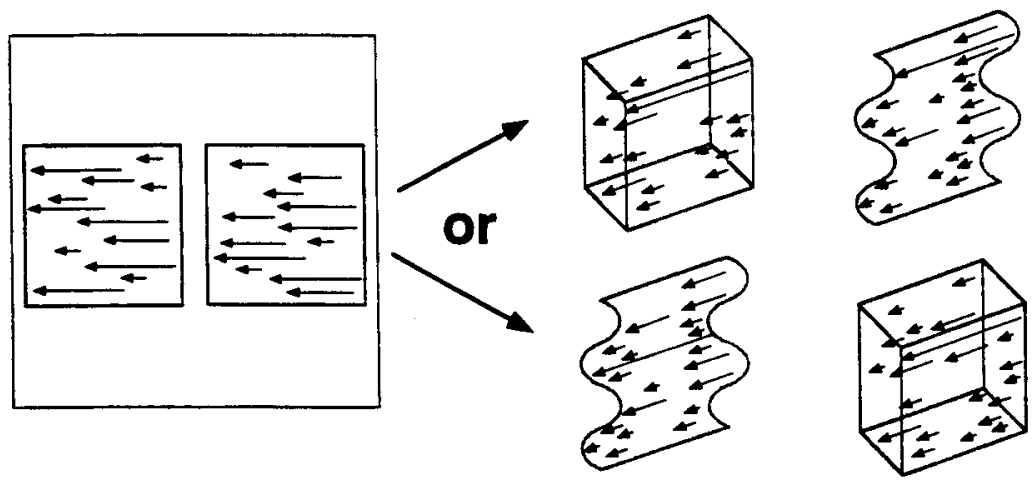

Figure 4. A graphic representation of the priming procedure used in Experiment 2. The left represents the screen as viewed by the observer. The right represents the type of information presented in the display. In Phase 1 , the velocity vectors would be absent for the no-prime case. The procedure was the same for Experiment 3 except that the motion in Phase 1 simulated an orthographic rotation rather than a perspective translation.

domized across subjects. There were 32 trials per condition per subject for a total of 256 trials per session. Prior to the start of each session, the subjects were shown flow fields that simulated each of the four corrugated surfaces (two frequencies and two amplitudes) for $8 \mathrm{sec}$ at a density of $7.95 \mathrm{dots} / \mathrm{deg}^{2}$.

\section{Results and Discussion}

The mean proportion correct for each subject for each condition was calculated and analyzed in a $2 \times 2 \times$ $2 \times 3$ ANOVA. The main effect of frequency was significant $[F(1,7)=41.93, p<.01]$. The subjects had higher detection rates at low spatial frequencies (proportion correct of .83) than at high spatial frequencies (proportion correct of .65). The effect of density was also significant $[F(1,7)=41.93, p<.01]$. The subjects had higher detection rates at the high texture density (proportion correct of .78) than at the low texture density (proportion correct of .70). The effect of prime type was also significant $[F(2,14)=4.19, p<.05]$. A post hoc comparison of the means for the three priming conditions using Tukey's HSD revealed that the subjects had significantly higher detection rates $(p<.05)$ for the surface prime condition (proportion correct of .79) than for either the motion prime condition (proportion correct of .72) or the no- 
prime condition (proportion correct of .72). The interaction between frequency and amplitude was also significant $[F(1,7)=7.83, p<.05]$. Performance was best in the low-frequency-high-amplitude condition (proportion correct of .85) than in all other conditions. Figure 5 presents the effect of prime type for each type of surface.

The contribution of frequency, texture density, and frequency and amplitude to 3-D surface detection has been described previously (Andersen, 1996; Andersen \& Wuestefeld, 1993). The new finding from this experiment is that priming with a 3-D corrugated surface from optic flow aided in the detection of that surface in a 2 AFC paradigm. Priming with the random velocity display did not increase detectability above baseline performance (no prime). This finding lends support to the hypothesis that the mechanisms responsible for recovering the deformation of the flow field, and not the individual velocities of the display, were primed by the 3-D corrugated surface prime condition.

The present results are consistent with the interpretation that the effect of priming occurred at the level of an analysis of deformation rather than at the level of the detection of velocities in the display. However, one alternative explanation is that the present results were due to priming another level of analysis. For example, the improved detection performance for the 3-D surface prime might have been due to activation of a representation of the surface independent of the deformation of the display (see Figure 6). Previous research has demonstrated the effectiveness of priming a generic representation in object recognition tasks (e.g., Beiderman, 1987). We examined this interpretation in Experiment 3 by presenting the subjects with the 3-D surface prime specified by orthographic rotation rather than by perspective translation.

\section{EXPERIMENT 3}

The possibility that our results were due to priming of some representation of the 3-D surface rather than the deformation of the flow field can be tested by simply changing the type of motion used to derive the surface. Previous research (Braunstein \& Andersen, 1984) has demonstrated that orthographic rotations can provide information for the recovery of 3-D shape. More recently, Braunstein (1994) has suggested that structure from motion (3-D structure from orthographic rotations) should be considered separately from other types of analyses for the recovery of 3-D shape. These observations suggest that the perception of a 3-D surface may likely occur from different motion processing mechanisms-one concerned with 3-D shape recovery from optic flow (perspective projections), and another analysis concerned with 3-D shape recovery from structure from motion (orthographic projections)-with the output used for a generic representation of a 3-D surface. In Experiment 2, we examined a 3-D surface prime that was specified by optic flow. In Experiment 3 , we examined the effects of a 3-D surface prime that was specified by orthographic projection as a method of priming a 3-D surface representation independent of optic flow. A random prime condition comparable to the one in Experiment 2 was also examined.

In addition to these two prime conditions and a target surface prime condition, a fourth prime condition was examined in which the subjects were presented with a 3-D corrugated surface undergoing horizontal translation similar to that examined in Experiment 2. However, the corrugation frequency of the prime surface was different from the corrugation frequency of the target surface. Our previous modeling research with 3-D corru-

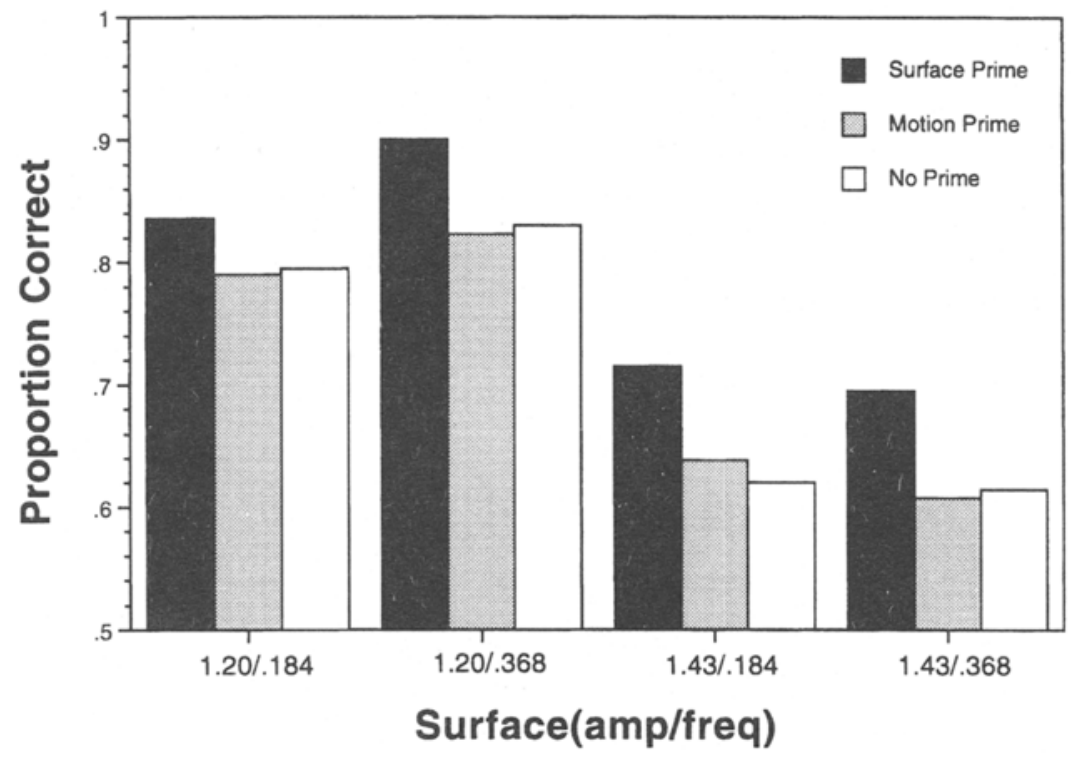

Figure 5. Mean proportion correct as a function of type of prime and surface (amplitude/ frequency) for Experiment 2. 


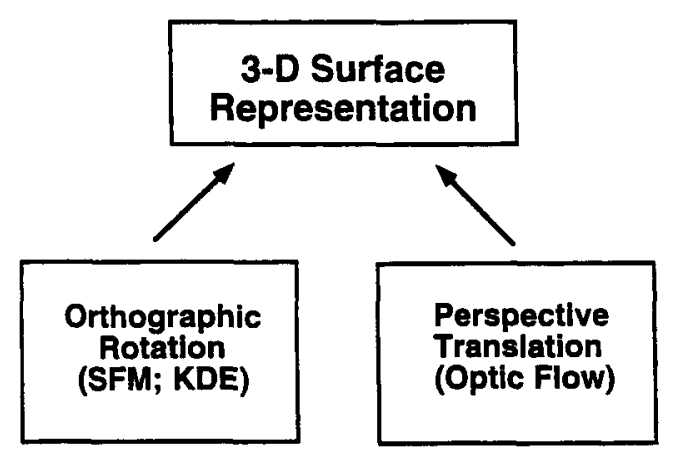

Figure 6. A graphic depiction of the separate contribution of orthographic rotation and perspective translation information to a 3-D surface representation.

gated surfaces from optic flow (Andersen, 1996; Andersen \& Atchley, 1997) indicates that different scales of shear detectors are optimal for surfaces with different corrugation frequencies. Specifically, surfaces with high corrugation frequencies require a smaller scale of shear detectors than surfaces with low spatial frequencies. This result is obvious since the greatest slant of a corrugated surface, specified by the peak-to-trough distance of the corrugated surface, occurs over a smaller spatial extent for high corrugation frequencies than for low corrugation frequencies.

This result suggests the following prediction: As the prime surface becomes more dissimilar from the target surface, due to a difference in corrugation frequency, the family of optimal shear detectors will also become more dissimilar. This should, in turn, result in less priming. We therefore examined a new priming condition in Experiment 4 in which the prime underwent the same motion (horizontal translation) as the target prime but was a different corrugation frequency.

To examine quantitative differences of deformation among the different surface prime displays, we derived the optimal deformation for each prime type. Domini, Caudek, and Proffitt (1997) present a derivation for deformation based on two views of three noncollinear points. Consider three points $\left(\mathrm{P}_{1}, \mathrm{P}_{2}, \mathrm{P}_{0}\right)$. According to their analysis, deformation (def) is defined as:

$$
\begin{aligned}
& \operatorname{def}=\frac{1}{|\sin \alpha|} \\
& \sqrt{\left(\frac{\left|V_{1}-V_{0}\right|}{\rho_{1}}\right)^{2}+\left(\frac{\left|V_{2}-V_{0}\right|}{\rho_{2}}\right)^{2}-2 \frac{\left|V_{1}-V_{0}\right|\left|V_{2}-V_{0}\right|}{\rho_{1} \rho_{2}} \cos \left(\alpha-\alpha_{\Delta}\right),}
\end{aligned}
$$

where $V_{0}, V_{1}, V_{2}$ are the velocity vectors of the projected points, $\rho_{1}$ and $\rho_{2}$ are the distances of the points $\mathrm{P}_{1}$ and $\mathrm{P}_{2}$ from $\mathrm{P}_{0}, \alpha$ is the angle between segments $\mathrm{P}_{0} \mathrm{P}_{1}$ and $\mathrm{P}_{0} \mathrm{P}_{2}$, and $\alpha_{\Delta}$ is the difference between the angles of the velocity vectors.
As discussed earlier, the optimal deformation for corrugated surfaces lies between the peak and trough of a single corrugation. This region corresponds to the greatest slant of the surface. Our calculations of deformation were thus derived using three points that defined an equilateral triangle with base and apex located at the peak and the trough of the corrugated surface, respectively. In Experiment 4, two target surfaces with different corrugation frequencies $(0.184$ and 0.368 cycles/deg) were examined. We thus calculated two sets of deformation values corresponding to the peak-to-trough distance of the two corrugation frequency surfaces. The peak-to-trough distance for the 0.184 - and 0.368 -cycles/deg corrugation frequencies were $2.71^{\circ}$ and $1.35^{\circ}$, respectively. The deformation values for the 0.184 -cycles/deg corrugation surface were 3.13 for perspective translation and 6.65 for orthographic rotation. The deformation values for the 0.368 -cycles/deg corrugation surface were 1.56 for perspective translation and 3.32 for orthographic rotation.

These analytic results indicate that the optimal deformation was quite different across the surface and motion conditions examined in Experiment 4 . For both corrugation surfaces, there was approximately a twofold increase in deformation for orthographic rotation, relative to perspective translation. There also was a twofold increase in deformation for the 0.368 -cycles/deg corrugation surface, relative to the 0.184 -cycles/deg corrugation surface for perspective translation.

\section{Method}

Subjects. The subjects were 8 psychology graduate and undergraduate students at the University of California, Riverside, who were naive with regard to the purpose of the experiment. Four subjects had participated in Experiment 3. The subjects were paid for their participation. All had normal or corrected-to-normal vision.

Apparatus. The apparatus was the same as that used in Experiments 2.

Design. The design was similar to that used in Experiment 2. A $2 \times 4$ within-subjects design was used. The independent variables were the spatial frequency of the sinusoidal surface $(0.184$ or 0.368 cycles/deg of visual angle) and the type of prime (a horizontally translating 3-D corrugated surface, a rotating 3-D corrugated surface, a random volume, or a horizontally translating 3-D corrugated surface of the opposite frequency of that of the target display).

Stimuli. The stimuli were similar to those used in Experiment 2 with the following additions. In addition to the priming displays examined in Experiment 2, we examined two priming displays. One prime consisted of an orthographic projection of the target 3 -D corrugated surface rotating about a vertical axis. In order to ensure that the perceived depth of the orthographic prime displays for the present experiment and Experiment 2 were equivalent, the depth extent of the rotating corrugated surface was perceptually matched to the perceived extent of the corrugated surface prime used in Experiment 2. A fourth prime type was added consisting of a horizontally translating 3-D corrugated surface of the opposite frequency as the target display. For example, if the target display had a spatial frequency of 0.184 cycles $/ \mathrm{deg}$, then the prime display had a spatial frequency of 0.368 cycles $/ \mathrm{deg}$. The amplitude ratio for the horizontal translation displays was 1.43 . The depth extent of the rotating corrugated surface was perceptually matched to the perceived extent of the corrugated surface displays. The den- 
sity of each target display was $0.16 \mathrm{dots} / \mathrm{deg}^{2}$. The density and corrugation frequency of the primes were identical to those used in Experiment 2.

Procedure. The procedure was identical to the procedure used in Experiment 2.

\section{Results and Discussion}

Data were analyzed using a $2 \times 4$ within-subjects ANOVA. For each surface type (frequency) and prime type, one score was calculated for each subject (the mean accuracy of the 32 individual trials), for a total of 8 data points per condition. The main effect of frequency was significant $[F(1,7)=9.205, p<.05]$. The subjects had higher detection rates at low spatial frequencies (proportion correct of .62) than at high spatial frequencies (proportion correct of .56). The main effect of priming was also significant $[F(1,7)=10.89, p<.01]$. A post hoc comparison (Tukey's HSD test) of the means for the four priming conditions revealed that the subjects had significantly higher detection rates $(p<.05)$ for the translation prime condition (proportion correct of .69) than for the rotation prime condition (proportion correct of .56), the no-prime condition (proportion correct of .52), or the opposite prime condition (proportion correct of .58). Neither the rotation prime condition nor the opposite prime condition was significantly different from the no-prime condition. The interaction between frequency and amplitude was not significant.

These results replicate the results obtained in Experiment 2 . Specifically, the results indicate increased detection performance for the translation prime condition, relative to the no-prime condition. No significant increase in detection accuracy occurred for the rotation prime condition, relative to the no-prime condition. The present results also indicate that significantly greater priming occurred for the translation prime than for the rotation prime condition and the opposite prime condition. This finding suggests that the increased performance for the translation prime is not simply the result of the presence of a surface during the priming part of the experiment.

\section{GENERAL DISCUSSION}

Three experiments were conducted to determine whether evidence of cooperativity exists for the detection of 3-D surfaces from optic flow. In Experiment 1, we used a hysteresis paradigm in which the texture density specifying a 3-D corrugated surface was either increased or decreased. The subjects' task was to press a key when the perception changed from random to surface (for ascending trials) or from surface to random (for descending trials). The threshold for a perceptual change was lower for descending trials-a result consistent with previous hysteresis studies of disparity and 2-D motion.

In Experiments 2 and 3, we used a priming paradigm to determine whether the hysteresis effect was the result of cooperativity at different levels of analysis. In Experiment 2, we used primes consisting of either the target
3-D corrugated surface or a random display (a randomly reordered corrugated surface flow field) to determine whether cooperativity was at the level of detection of individual velocities in the display or at the level of shear detectors purported to be responsible for detection of a 3-D corrugated surface. The results indicate that priming occurred only for the 3-D corrugated surface display, providing support for the hypothesis that cooperative mechanisms in 3-D surface detection from optic flow are present in the extraction of shear and not for the recovery of the individual velocities of the display. In addition, the results of Experiment 2 indicate that no significant difference occurred between the no-prime condition (mean accuracy of .72) and the random prime condition (mean accuracy of .72).

In Experiment 3, we compared priming with a prime undergoing perspective translation and a prime undergoing orthographic rotation as a method of determining whether the priming observed in Experiment 2 was due to the priming of a generic representation of the surface. We also used an opposite prime condition to determine whether the same set of shear detectors must be activated for priming to occur or whether priming can occur if a different set of shear detectors are activated. The results of Experiment 3 show that the similar translating case alone served as an effective prime.

The results of the three experiments, considered together, allow the following conclusions. First, we have provided evidence of a hysteresis effect for the detection of 3-D surfaces from optic flow. Second, evidence of cooperativity was found, using a priming paradigm, for the two shear components of deformation. The results of Experiment 2 did not support the hypothesis that cooperativity occurred for the detection of individual velocities in the display. In addition, no evidence was found from Experiment 3 to support the hypothesis that the results of Experiment 2 were due to the priming of a generic representation of the surface.

There are two possible alternative explanations that one might argue account for the results of the present experiments. One argument is that the results were due to attentional issues. Another argument is that the results were due to preactivation of the spatial distributions of velocities. We will consider both of these possibilities separately. The first alternative explanation is that the results were due to the structured target serving as an attentional cue, providing the subjects with a clear perception of the appearance of the target. If this hypothesis is correct, then providing the subjects with the orthographic rotation surface prime should also have resulted in a priming effect, since this prime condition provided the subjects with a clear perception of the appearance of the target surface. The results of Experiment 3 indicate that detection performance following the orthographic target prime was significantly lower than the detection performance of the horizontally translating target prime and was not significantly different from the no-prime condition. One might argue that this hypothesis is still valid if the rotating 3-D 
corrugated surface prime was not detectable. However, the display conditions selected for the orthographic rotation condition were well above threshold (Turner, Braunstein, \& Andersen, 1997). Thus, the results do not support the conclusion that the priming effects in Experiments 2 and 3 were the result of attention.

The second alternative explanation is that the priming was due to a preactivation of the spatial distribution of velocities important in detecting the target display. In Experiments 2 and 3, the spatial distributions of velocities used for the target were not randomly sampled from the set of velocities used to define a specific priming display. Instead, the set of velocities was independently se- lected from the total set of velocities used to define the surface. The probability that the spatial distribution of velocities in the target display was identical to a subset of the spatial distribution of velocities in the priming display can be calculated by determining the probability of all of the velocities (points) in the surface display would have the identical random locations in the random display. This probability (determined by calculating the probability of any given dot randomly located at a particular pixel location occupying the same pixel location in second random sample) was .0000016 . This calculation suggests that it is unlikely that the priming effect in Experiments 2 and 3 were due to the priming of the specific
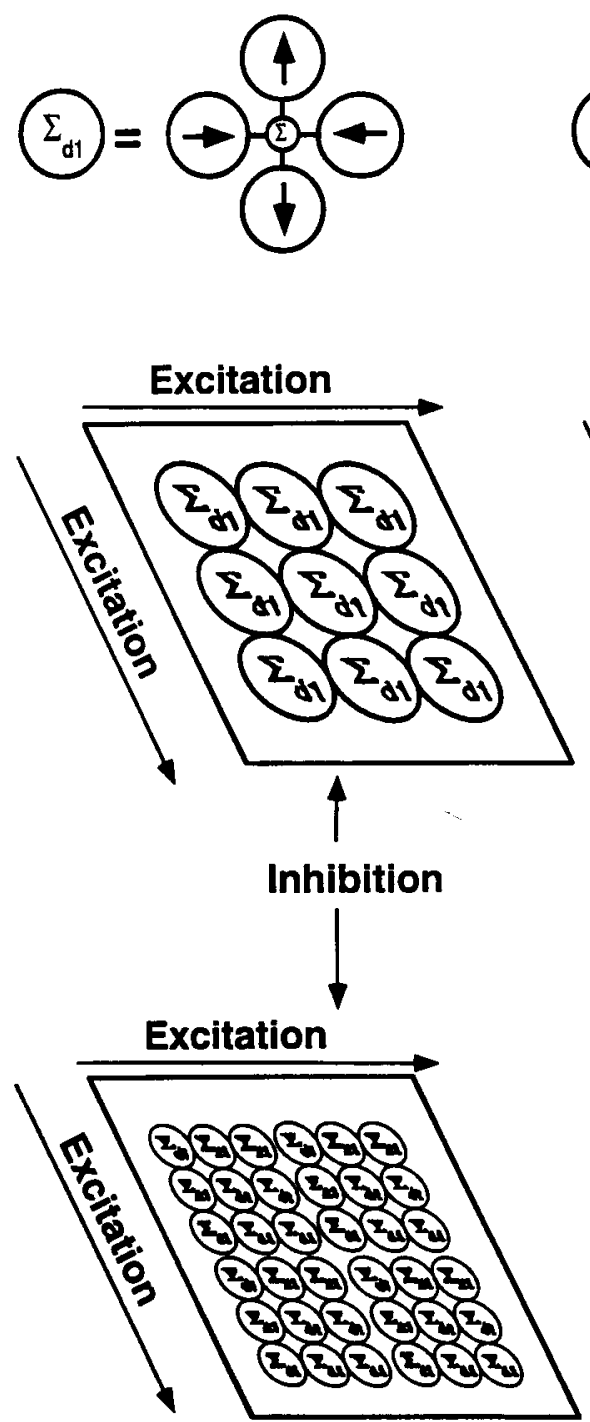
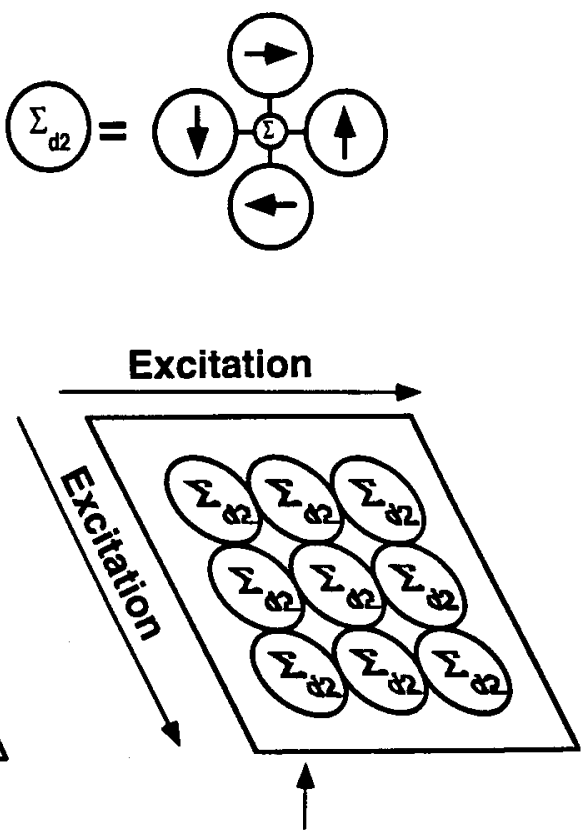

Inhibition

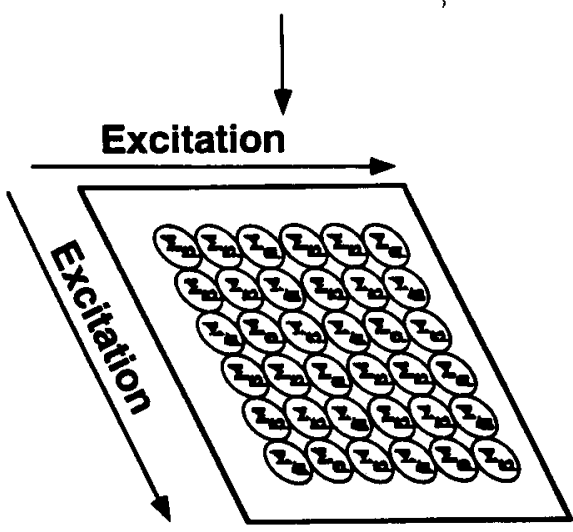

Figure 7. A model of surface detection from optic flow using multiple layers of cooperative shear detectors. $\Sigma_{\mathrm{d} 1}$ and $\Sigma_{\mathrm{d} 2}$ refer to the two types of shear detectors. The illustration depicts two different families of shear detectors (illustrated on the left and the right side of the figure) and two layers of mechanisms (illustrated in the middle and the bottom of the figure). The two layers of shear detectors respond optimally to different velocity magnitudes. According to the model, cooperativity occurs within each layer, and inhibition occurs between the layers. 
spatial distribution of velocities used to define a specific target display.

In addition to the results reported from the present experiments, previous empirical results (Andersen, 1996; Andersen \& Atchley, 1995; Andersen \& Wuestefeld, 1993) have provided indirect evidence of cooperativity. In these studies, the ability to detect single-sine wave and complex sine-wave corrugated surfaces were examined. A consistent result across these studies was that detection performance was much lower for complex corrugated sine-wave surfaces than for single sine-wave corrugated surfaces. One explanation that may account for this result is the manner of cooperative connections among shear detectors. There may exist a specific class of shear detectors that are optimal, in terms of spatial sampling and velocity magnitude, for the detection of a single sine wave of a specific amplitude and frequency. A lattice of such detectors might be networked together in a cooperative manner, resulting in greater output than lattices of detectors that are optimal for surfaces of a different corrugation amplitude and frequency. In contrast, detection of a complex sine-wave corrugated surface might have a set of optimal shear detectors (optimal in terms of spatial sampling and velocity magnitude) based on the underlying prime frequencies of the corrugated surface. If the shear detectors are networked such that detectors of similar scale (spatial size and velocity magnitude) are cooperative while detectors of dissimilar scale are inhibitory, then one would expect greater detectability for single corrugated surfaces than for complex corrugated surfaces (see Figure 7).

The results of our previous studies on the detection of single and complex corrugated surfaces, along with the results of the present study, suggest that the cooperativity of surface detection mechanisms is an important consideration for computational modeling of surface detection from optic flow. Previous research (Andersen, 1996; Andersen \& Atchley, 1995) concerned with 3-D surface detection from optic flow has considered analyses based on the two shear components of deformation. According to the model, a lattice of shear detectors was used to compute the absolute summed value of the two shear components. The analyses considered in our previous model did not incorporate cooperativity among neighboring regions. Instead, the output within local regions was simply summed together to produce a composite average shear value for each condition. One possible cooperative scheme would be to add inhibitory connections between layers of shear detectors tuned to different velocity characteristics and to add excitatory connections within a layer of shear detectors (see Figure 7). An important goal of future research will be to further consider cooperativity in modeling shear detectors for 3-D surface detection.

In summary, the present results provide evidence of cooperativity in the detection of 3-D surfaces from optic flow. Using a priming paradigm, our results suggest that the cooperativity observed in the present experiments was the result of cooperative interactions among shear detectors rather than cooperative interactions among 2-D motion detectors or cooperativity of a generic representation of 3-D surfaces. These results, taken together with the results of empirical studies on the detection of simple and complex corrugated surfaces, suggest that surface detection models of optic flow should include cooperative interactions among shear detectors of similar scale.

\section{REFERENCES}

ANDERSEN, G. J. (1996). Detection of smooth three-dimensional surfaces from optic flow. Journal of Experimental Psychology: Human Perception \& Performance, 22, 945-957.

ANDERSEN, G. J., \& ATChLEY, P. (1995). Age-related difference in the perception of 2D and 3D motion perception. Psychology \& Aging, 10, 650-658.

ANDERSEN, G. J., \& ATChley, P. (1997). Smoothness of the velocity field and three-dimensional surface detection from optic flow. Perception \& Psychophysics, 59, 358-369.

Andersen, G. J., \& Wuestefeld, A. P. (1993). Detection of threedimensional surfaces from optic flow: The effects of noise. Perception \& Psychophysics, 54, 321-333.

BEIDERMAN, I. (1987). Recognition-by-components: A theory of human image interpretation. Psychological Review, 94, 115-147.

Biederman, I., \& COOPER, E. E. (1991). Priming contour-deleted images: Evidence for intermediate representations in visual object recognition. Cognitive Psychology, 23, 393-419.

Braunstein, M. L. (1994). Structure from motion. In E. T. Smith \& R. J. Snowden (Eds.), Visual detection of motion (pp. 367-394). New York: Academic Press.

Braunstein, M. L., \& Andersen, G. J. (1981). Velocity gradients and relative depth perception. Perception \& Psychophysics, 29, 145-155.

Braunstein, M. L., \& Andersen, G. J. (1984). Shape and depth perception from parallel projections of three-dimensional motion. Journal of Experimental Psychology: Human Perception \& Performance, 10, 749-760.

Braunstein, M. L., Hoffman, D. D., \& Pollick, F. E. (1990). Discriminating rigid from nonrigid motion: Minimum points and views. Perception \& Psychophysics, 47, 205-214.

BRaunstein, M. L., \& PAYNE, J. W. (1969). Perspective and form ratio as determinants of relative slant judgments. Journal of Experimental Psychology, 81, 584-590.

Braunstein, M. L., \& Tittle, J. S. (1988). The observer-relative velocity field as the basis for effective motion parallax. Journal of Experimental Psychology: Human Perception \& Performance, 15, 582 590

Chang, J., \& Julesz, B. (1984). Cooperative phenomena in apparent motion perception of random-dot cinematograms. Vision Research, 24, 1781-1788.

Chiappe, D. L., \& Macleod, C. M. (1995). Negative priming is not task bound: A consistent pattern across naming and categorization tasks. Psychonomic Bulletin \& Review, 2, 364-369.

De Bruyn, B., \& Orban, G. A. (1990). The importance of velocity gradients in the perception of three-dimensional rigidity. Perception, 19, 21-27.

Domini, F., Caudek, C., \& Proffitt, D. R. (1997). Misperceptions of angular velocities influence the perception of rigidity in the kinetic depth effect. Journal of Experimental Psychology: Human Perception \& Performance, 23, 1111-1129.

Feldman, J. A., \& Ballard, D. H. (1982). Connectionist models and their properties. Cognitive Psychology, 6, 205-254.

Fender, D., \& Julesz, B. (1967). Extension of Panum's fusional area in binocularly stabilized vision. Journal of the Optical Society of America, 37, 819-830.

Fox, E. (1995). Negative priming from ignored distractors in visual selection: A review. Psychonomic Bulletin \& Review, 2, 145-173.

Gibson, J. J. (1966). The senses considered as perceptual sistems. Boston: Houghton Mifflin.

Helmholtz, H. L. F. voN (1962). Treatise on physiological optics 
(Vol. 3, J. P. C. Southall, Ed. \& Trans.). New York: Dover. (Original work published 1867)

JuLESZ, B. (1971). Foundations of cyclopean perception. Chicago: University of Chicago Press.

JULESZ, B. (1983). Commentary on Grossberg, S., The quantized geometry of visual space: The coherent computation of depth, form, and lightness. Behavioral \& Brain Sciences, 6, 625-692.

KoENDERINK, J. J. (1986). Optic flow. Vision Research, 26, 161-180.

KOENDERINK, J. J., \& VAN DOORN, A. J. (1977). How an ambulant observer can construct a model of the environment from geometric structure of the visual inflow. In G. Hauske \& E. Butendent (Eds.), Kibernetic (pp. 235-252). Munich: Oldenborg.

Lagae, L., MaEs, H., Raiguel, S., Xaio, D. K., \& Orban, G. A. (1994). Responses of macaque STS to optic flow components: A comparison of areas MT and MST. Journal of Neurophysiology, 71, 15971626.

LaWson, R., Humphreys, G. W., \& Watson, D. G. (1994). Object recognition under sequential viewing conditions: Evidence for viewpointspecific recognition procedures. Perception, 23, 595-614.

Longuet-Higgins, H. C., \& PRAzdny, K. (1980). The interpretation of a moving retinal image. Proceedings of the Royal Society of London: Series B, 208, 385-397.

MaY, C. P., KANE, M. J., \& HASHER, L. (1995). Determinants of negative priming. Psychological Bulletin, 118, 35-54.

MCNamara, T. P. (1992). Theories of priming: I. Associative distance and lag. Journal of Experimental Psychology: Learning, Memory, \& Cognition, 18, 1173-1190.

Nawrot, M., \& Sekuler, R. (1990). Assimilation and contrast in motion perception: Explorations in cooperativity. Vision Research, 30, 1439-1451.

Norman, J. F., \& Lappin, J. S. (1992). The detection of surface curva- tures defined by optical motion. Perception \& Psychophysics, 51, 386-396.

Reingold, E. M., \& Goshen-GotTSTEIN, Y. (1996). Separating consciously controlled and automatic influences in memory for new associations. Journal of Experimental Psychology: Learning, Memory, \& Cognition, 22, 397-406.

Rogers, B. \& Graham, M. (1979). Motion parallax as an independent cue for depth perception. Perception, 8, 125-134.

ROGERS, S., \& ROGERS, B. J. (1992). Visual and nonvisual information disambiguate surfaces specified by motion parallax. Perception \& Psychophysics, 52, 446-452.

TENPENNY, P. L. (1995). Abstractionist versus episodic theories of repetition priming and word identification. Psychonomic Bulletin \& Review, 2, 339-363.

ToDD, J. T. (1984). The perception of three-dimensional structure from rigid and nonrigid motion. Perception \& Psychophysics, 36, 97-103.

Turner, J., Braunstein, M. L., \& ANDERsen, G. J. (1997). Relationship between binocular disparity and motion parallax in surface detection. Perception \& Psychophysics, 59, 370-380.

VERrI, A., Girosi, F., \& TORRE, V. (1990). Differential techniques for optical flow. Journal of the Optical Society of America A, 7, 912922.

Williams, D., \& Phillips, G. (1987). Cooperative phenomena in the perception of motion direction. Journal of the Optical Society of America A, 4, 878-885.

Williams, D., Phillips, G., \& Sekuler, R. (1986). Hysteresis in the perception of motion direction as evidence for neural cooperativity. Nature, 324, 253-254.

(Manuscript received January 7,1997 revision accepted for publication August 19, 1997.) 